sans ếr ə le mins du mond a attirés, et mêmè ils pauvent, si leur vitesse de déplacement est différente, de nouveau s'échapper l'un de l'autre après s'être heurtés, de sor'e que pas une fois l'agglutination n'est spécialement vigoureuse. Dans ces conditions, il est évident que la montée de la crème se réalise plus facilement, si les globules de matière grasse ont plus fréquemment l'ozeasion de se heurter, c'est-à-dire plus ils sont serrés. Par contre, si la montée de la crème a d'abord commencé, les globules de matière grasse se rassembleront d'eux-mêmes de plus en plus, parce que tous se rassemblent vers le haut, et la teneur originale du lait en matière grasse a de moins en moins d'importance. Une trop forte teneur en matière grasse au début est même, comme on le sait par la crème grasse, un empêchement au déplacement des globules de matière grasse.

Quand j'ai commencé ce travail, je pensais aussi, d'accord avec les opinions admises jusqu'ici, que la vitesse avec laquelle la montée de la crème a vait lieu dépendait essentiellemert de la grosseur des globules de matière grasse et de la viscosité du plasma du lait. Nous avons vu maintenant qu'il faut des agrégats de globules de matière grasse pour avoir une montée de crème rapide, et nous avons apporté plusieurs preuves de ce que la viscosité du plasma du lait est d'importance secondaire à cet égard. Toutefois, la meilleure preuve est peut-être que de l'eau pure, à laquelle on ajoute une certaine proportion de matière grasse par addition de crème concentrée, ne laisse pas monter de crème. Ceci est réellement très remarquable et peut provenir de la structure caractéristique des globules de matière grasse du lait, car l'eau pure est plutôt un mauvais émulsionnant, pour la matière grasse. Par eontre, on obtient une abondante montée de crème aussitôt qu'on ajoute à l'eau un peu de lait cru ou de sérum cru.

(A suivre)

\title{
DES AVANTAGES DE LA FILTRATION SUR LE NETTOYAGE MÉCANIQUE DU LAIT AU MOYEN D'ESSOREUSES CENTRIFUGES
}

par H. STASSANO et A. P. ROLLET.

On cherche à débarrasser le lait des impuretés qui y pénètrent dès sa sortie du pis de la vache - quelques-unes même sont entraînées par lui à partir des trayons - tout d'abord, à la ferme, par un simple tamisage, assez suffisant pour retenir les grosses souillures provenant de la litière, de la pəau de la vache, poils, croûtes de bouze desséchées ; ensuite, à la laiterie de ramassage dans laquelle le lait des différentes fermes d'une même localité ou de plusieurs contrées voisines, est groupé pour être, dans la plupart des cas, pasteurisé avant son transport aux centres de consommation, plus ou moins éloignés des lieux de próduction, (jusqu'à 500 kilomètres aujourd'hui pour le lait introduit à Paris); ce premier nettoyage est parachevé au moyen d'une filtration plus sévère, sur plusieurs couches de tarlatane, une étoffe en coton, assez légère 
et claire, ou sur plusieurs feuilles de ouats hydrophile laissant passer également sans pression et sans arrêt le lait.

Dans les laiteries mieux outillées, on utilise surtout pour ce second et plus rigoureux nettoyage, un moyen mécanique. On fait passer le lait par des essoreuses tournant à une assez grande vitesse, au moins mille tours à la minute. Sur les parois de ces engins, particulièrement adaptées à les recevoir, à les garder, sous l'impulsion incessante de la force centrifuge, s'amassent ou devraient s'amasser les impuretés de toute sorte dont on doit débarrasser le lait.

A première vue; quand au bout de plusieurs heures de travail de ces machines, par lesquelles 2 à 3 dizaines de milliers de litres de lait ont passé, on les démonte et on regarde l'épaisse et dense boue couleur gris qui se trouve accumulée, accrochée sur leurs parois, on a l'impression que le but désiré a été parfaitement atteint.

Mais si, au lieu de s'arrêter à cette première impression, on se met à examiner de près cette boue, comme nous l'avons fait, sachant à bon escient ce dont est capable la force centrifuge lorsqu'elle agit sur des corpuscules de l'ordre de grandeur et de densité des résidus cellulaires et des microbes, on trouve que cette boue est constituée essentiellement de terre : pas la moindre trace d'accumulation en elle de ces éléments organiques - cellules, épithéliales, leucocytes ou leurs résidus, microbes - qu'il intéresse le plus de séparer, pour contribuer par ce moyen mécanique à l'assainissement du lait.

L'analyse chimique que nous avons faite de'cette boue vient à l'appui de ce qui a été trouvé par l'examen au microscope. La proportion insignifiante de l'azote par rapport à la masse du produit sec démontre irréfutablement l'inexistence de la moindre séparation des déchets cellulaires et des microbes, que les partisans de l'emploi des essoreuses centrifuges escomptent aveuglément et, nous ajouterons, en vrais ignorants. Car pour peu que l'on soit habitué aux manipulations de physiologie ou de biologie générale, on sait que pour séparer, par exemple, les éléments figurés du sang du plasma sanguin rendu incoagulable, les leucocytes au sein d'un exsudat, les microbes de leur bouillon de culture, il faut plus d'une heure avec une centrifuge du genre de celles de Rhun (les plus perfectionnées il y a trente ans) accomplissant 1.200 tours à la minute; avec celles plus récentes du type Leune, effectuant 4 à 5 mille tours à la minute, il faut au moins 5 minutes et cela, en appliquant, dans les deux cas, la force centrifuge pendant toute la durée de l'opération sur les mêmes corps en suspension dans une même colonne liquide immobile qui ne dépasse pas 15 centimètres de hauteur. Comment pourrait-on prétendre alors, même en employant les centrifuges américaines faisant de 18 à 20.000 tours à la minute, obtenir la moindre séparation, agir sur les impuretés organiques cे éliminer, dans une veine liquide mouvante, à allure torrentueuse, tel que le lait pénétrant 
par le haut dans une de ces essoreuses en question, au débit de 4 à 5 mille litres par heure?

ANALYSE DE LA BOUE RECUETLLIE SUR LES PAROIS D'UNE DES ESSOREUSES de la lafterie Centrale de Strasbourg, faisant 1.200 TOURS A LA MINUTE APRÈS LE NETTOYAGE DE 25 A 30 MILLE LITRES DE LAIT.

\section{Humidité}

Il se dégage à l'étuve à $105^{\circ}$ une odeur nauséabonde.

Durée de l'opération : 10 heures.

Humidité moyenne . . . . . . . . . . . $69,7 \%$

Produit see. . . . . . . . . . . . $30,3 \%$

Cendres

Traitement au four à moufle, moyenne $7 \%$.

Le produit see donne $7 \%$ de cendres.

Azote

Traitement du produit see au Kjeldahl.

Résultat des deux opérations :

$1,42 \%$. . . . 1,20\% Soit : Azote 1,35\% du produit sec

Il est incontestable que pour débarrasser le lait cru de la terre qui le souille en proportion variable, selon le degré de propreté des pots à lait dans les différentes fermes, l'essorage mécanique par la force centrifuge, convient le mieux. Ces essoreuses sont d'une marche très régulière et sont très faciles à nettoyer, l'opération finie.

Les filtres, par contre, quelqu'ils soient, peuvent laisser échapper un peu de cette terre, le tissu dont ils sont constitués est trop clair, il s'y produit des petits trous qui peuvent passer inaperçus. De plus les filtres se colmatent assez promptement; on doit les changer au moins toutes les deux à trois heures, alors que les essoreuses peuvent fonctionner deux ou trois fois plus longtemps sans besoin d'arrêt.(1)

Voici maintenant, d'après nos propres observations, le principal, nous dirons même l'unique désavantage de l'emploi des essoreuses en question dans les laiteries.

Déjà, avant nos analyses, on avait remarqué que le lait prend de l'air, forme de la mousse, en tourbillonnant à l'entrée des essoreuses.

(1) Dans le filtre adopté par l'un de nous, pour ses pasteurisateurs de lait en couche mince, le lait traverse successivement deux étamines de soie, en usage dans la meunerie le $n^{0} 44$ d'abord, le $n^{0} 66$, à fils très rapprochés, ensuite; enfin plusieurs couches de tarlatane. Pour éviter les trous, il faut supprimer les coutures dans ces tissus. On y arrive en retenant les extrémités, comme dans des étaux, par des cadres métalliques (cuivre étamé) s'appliquant l'une sur l'autre.

A la station gouvernementale danoise d'essais en laiterie d'Hillerod où un pasteurisateur Stassano fonotionne depuis plus d'un an, le directeur, Mr. Hansen a remplacé avee avantage les étamines par des flanelles.

Il faut, en outre, pouvoir régler le courant de manière à être sîr qu'il n'y ait pas de lait qui déborde. 
Or la mousse du lait augmente l'irrégularité de l'action du chauffage sur les microbes, irrégularité déjà par elle-même assez importante dans les pasteurisateurs usuels.

Il est vrai qu'on a diminué la valeur, la portée de cette irrégularité d'action des pasteurisateurs usuels, tous du type danois, en les employant autrement. Lans ce nouveau mode d'emploi, qui constitue ce que l'on appelle la pasteurisation basse le lait est monté plus rapidement dans le pasteurisateur, il atteint par conséquent à la sortie une température inférieure $-65^{\circ}$ - à celle, environ $85^{\circ}$, qu'il prend dans le mode d'emploi usuel; mais au lieu d'être refroidi immédiatement, comme dans le procédé usuel, le lait est maintenu chaud, à $63^{\circ}$, dans des bassins appropriés dits chambreurs en français, holders en anglais.

Or, si en maintenant chaud le lait dans ces bassins quelques 25 à 30 minutes, on parvient à remédier, en partie tout au moins, à l'irrégularité de l'action des parois chauffantes des pasteurisateurs, irrégularité d'action augmentée par la présence de la mousse, cette même mousse provenant de l'essoreuse et que le lait entraîne toujours à travers le pasteurisateur jusqu'aux chambreurs, aux "holders ", apporte dans ce nouveau procédé de pasteurisation une autre cause de trouble, à savoir : selon la proportion plus ou moins forte de la mousse, d'après l'avis de différents auteurs, la montée spontanée de la crème est plus au moins gênée.

Il s'ensuit que la mousse engendrée par les essoreuses si elle ne nuit pas d'une manière, nuit d'une autre, mais elle nuit toujours, qu'il s'agisse de pasteurisation haute ou de pasteurisation basse.

La mousse done a été reconnue avant nous comme un défaut des essoreuses; nos analyses, concernant les gaz et l'équilibre chimique du lait cru, avant et après l'essorage, nous ont fait pénétrer plus intimement dans la nature du trouble en question causé par l'emploi des essoreuses comme moyen de nettoyage du lait cru.

D'après nos analyses, le lait à la suite du passage par l'essoreuse perd une certaine quantité d'acide carbonique, et fixe, d'autre part, une petite quantité d'oxygène appartenant à l'air qui se trouvait déjà dissout dans le lait et qui s'y dissout même au moment de l'essorage, d'où résulte la proportion relativement plus forte, par rapport au volume trouvé, de l'azote et des gaz résiduaires suivant notre expérience du 4 avri] 1924 .

Nous communiquons en détail dans le petit tableau suivant les résultats de cette opération, choisie parmi les autres, à titre d'exemple.

La lecture de ce tableau est assez facile ; la perte de l'acide carbonique - $1,055 \mathrm{ce}$. - monte à plus d'un centimètre cube par $100 \mathrm{cc}$. de lait : c'est-à-dire elle est la valeur d'un quart environ du taux normal de l'acide carbonique dans le lait cru. On y constate en outre, une certaine disparition de l'oxygène, vraisemblablement fixé par le lait circulant, 
ANALYSE VOLUMÉTRIQUE COMPARATIVE CORRESPONDANT

A 100 CC. DE LAIT,

\begin{tabular}{l|c|c|c|c|c}
\hline \multicolumn{1}{c|}{ Nature du Lait } & $\begin{array}{c}\text { Acidité } \\
\text { en degrés } \\
\text { Soxhlet }\end{array}$ & $\begin{array}{c}\text { Volume } \\
\text { total } \\
\text { du gaz }\end{array}$ & $\begin{array}{c}\text { Volume } \\
\text { acide } \\
\text { carbonique }\end{array}$ & $\begin{array}{c}\text { Volume } \\
\text { oxygène }\end{array}$ & $\begin{array}{c}\text { Azote } \\
\text { et gaz } \\
\text { résiąuaires }\end{array}$ \\
\hline & & c.e. & c.c. & e.e. & c.e. \\
Lait cru avant l'essorage & 70,2 & 6,40 & 4,495 & 0,50 & 1,405 \\
Même lait après l'essorage & 70,0 & 5,44 & 3,440 & 0,45 & 1,55
\end{tabular}

alors que le volume de l'azote et des gaz résiduaires augmente après l'essorage: ce qui est la preuve manifeste de l'aération du lait accompagnée de l'oxydation telle qu'elle apparaît de la comparaison des deux valeurs trouvées pour l'oxygène, avant et après l'essorage.

Pour terminer, nos recherches apportent en plus d'une nouvelle démonstration de l'aération dont souffre le lait à la suite de l'essorage par la force centrifuge, aération qui se traduit par un certain degré d'oxydation nuisible au lait autant que la formation concomitante de la mousse déjà déplorée - la constatation d'une perte assez importante en acide carbonique dans le lait qui vient d'être nettoyé au moyen de ces appareils.

Or, si cette perte pout être envisagée comme indifférente dans la pratique des procédés usuels de pasteurisation au cours desquels le lait subit des partes enoore plus importantes de ce même gaz qui est un des éléments les plus précieux de sa constitution chímique, il n'en est pas de même pour le procédé préconisé et mis au point industrielle. ment par l'un de nous, le.procédé de la couche mince.

Par ce procédé; l'équilibre chimique du lait est respecté. Avec la conservation intégrale de l'acide carbonique, la solubilité des sels de chaux et des phosphates demeure par conséquent intacte dans le lait traité par ce procédé.

On prétend encore qu'en faisant passer le lait par les essoreuses, on parvient à rompre les chaînettes que certains microbes, les coccus, forment lorsqu'ils se développent assez rapidement : dans ce cas, par l'essorage, on faciliterait l'action du chauffage sur les microbes ainsi détachés, isolés. En premier lieu, en fait de microbes de lait formant des chaînettes, il n'y a guère que les streptocoques lactis qui les engendrent, à condition toutefois que la température soit assez élevée pour déterminer une intense fermentation lactique : ce qui se produit déjà à la température environ de l'étuve à $37^{\circ}$. Deuxièmement, on n'a pas à s'occuper à cet égard des microbes pathogènes, car leur nombre demeure toujours très restreint dans un lait qui, quoique contaminé, garde l'aspect de lait normal.

Pareil avantage des essoreuses, en tout cas, ne peut être envisagé 
que comme une pure hypothèse, basée sur une vue de l'esprit aussi simple qu'inexacte. Car comment auraient fait les partisans de cette hypothèse pour s'assurer de sa véracité, e'est-à-dire, de l'augmentation du nombre des microbes du lait pendant la courte traversée de l'écrémeuse ? Ils auraient pu avoir recours, comme d'habitude, au dénombrement par le procédé des ensemencements sur milieux solides en plaques de Pétri. Or, pour réaliser ees ensemencements avee du lait cru, on est obligé au préalable d'opérer de 4 à 5 dilutions successives de l'échantillon de lait en examen, dans 4 à 5 tubes à essais, renfermant chacun neuf fois le volume, en bouillon stérilisé, du volume de la dilution précédente, soit un centimètre cube. Et à chaque nouvelle dilution, pratiquée, bien entendu, à l'aide d'une nouvelle pipette graduée, stérilisée, on rince vivement la dite pipette avec le nouvel échantillon de bouillon et le mélange est fortement secoué pour pouvoir prélever, pour la dilution suivante, un centimètre cube d'une émulsion microbienne parfaitement homogène. Bref, dans ces 4 à 5 dilutions successives, les microbes renfermés dans l'échantillon de lait prélevé au début, sont beaucoup plus secoués que dans le simple essorage dont il est question; les chaînettes des streptocoques lactis, s'il y en a, peuvent, par conséquent, être brisées aussi bien pendant l'essorage qu'après, c'est-àdire pendant les opérations préparatoires du dénombrement des microbes que l'hypothèse suppose augmentés.

Pourtant il se produit effectivement un surcroît d'activité au sein des ferments lactiques dans le lait qui traverse une essoreuse en action. On peut s'en assurer d'une manière toute autre, mais à laquelle les partisans de la théorie en question n'ont pas songé. La voici : on prélève aseptiquement un échantillon du lait cru sur lequel on opère, au moment même où il pénètre dans l'essoreuse, et un second échantillon, avec les mêmes présautions d'asepsie, immédiatement après sa sortie de l'appareil. Les deux échantillons, recueillis dans des récipients parfaitement stériles, sont exposés à l'étuve. Le titrage de leur respective acidité, en degré Soxhlet, est pratiqué simultanément avant le séjour à l'étuve et quelques heures après. Les résultats de quelques-uns de ces essais sont les suivants :

Essai du 11 juin 1924:

Après 8 heures de séjour à l'étuve à $37^{\circ}$ :

Acidité du lait cru avant l'essorage : $10^{\circ}, 4$.

Acidité du mème lait après l'essorage : $11^{\circ}, 6$.

Essai du 18 juin 1924:

10 Lait cru avant l'essorage: $7^{\circ}, 7$.

$2^{\circ}$ Même lait après l'essorage : $7^{\circ}, 4$.

Cette légère chute d'acidité est en rapport avec la perte en acide carbonique subie par le lait pəndant l'essorage. 
Ces deux échantillons $1^{\circ}$ et $2^{\circ}$ accusaient respectivement après 24 heures de séjour à l'étuve :

$1^{0}$ Le lait non essoré : $34^{\circ}, 6$.

2 . Le même lait essoré : $35^{\circ}, 2$.

Essai du 19 juin 1924 :

10 Lait eru avant 1'essorage : 70,4.

$2^{\circ}$ Même lait après essorage : $7^{\circ}, 0$.

Après 8 heures de séjour à l'étuve respectivement :

10 Lait avant essorage : $17^{\circ}$.

$2^{\circ}$ Lait après essorage : $17^{\circ}, 8$.

On ne peut done douter que se produit, ou, pour être plus précis, que s'amorce au cours de l'essorage, une légère augmentation du nombre des mierobes véhiculés par le lait cru. Cette plus forte poussée microbienne, portant naturellement sur les ferments lactiques, doit se rattacher, très vraisemblablement, à la très sensible aératìon, accompagnée de fixation d'oxygène, que le lait subit dans cette opération, et qui est présisément l'un des inconvénients de l'essorage centrifuge dont nous venons de parler.

\section{LA VITAMINE C DANS LE LAIT CONCENTRÉ SUCRÉ}

\section{par Dr A. BAKKE}

Chef des Services Scientifiques de la Nestlé \& Anglo-Swiss Condensed Milk Co.,

Assisté par

Mile V. ASCHEHOUG,

Assistante au Laboratoire Central de la N. \& A. S. C. M. Co à Vevey.

\section{(Suite et fin)}

Nous avons démontré dans un précédent article que la vitamine $C$ du lait frais résiste parfaitement au eours de la fabrieation du lait con zentré sucré, lorsque celle-ci se fait dans de bonnes conditions.

En outre, nous avons établi que les propriétés antiscorbutiques du lait frais se conservent longtemps dans le lait concentré sucré.

Il était intéressant de vérifier que la vitamine $\mathrm{C}$ du lait concentré sucré reste active pendant une période plus longue encore que la période envisagée lors des premiers essais. Des travaux ont été faits en France dans cet ordre d'idées il y a quelques années, mais les relations qui en ont été publiées sont trop peu détaillées pour qu'il soit possible de suivre de près la marche des observations. Il va sans dire qu'il est plus facile d'obtenir des précisions de ce genre dans notre laboratoire, où l'on utilise et examine les produits de toutes les fabriques Nestlé que notre Service scientifique a pour mission de eonseiller.

Lors de nos premières expériences, nous avions réservé, afin de l'examiner plus tard, une partie du lait concentré sucré utilisé, de sorte que la nouvelle série d'expériences dont nous allons dire quelques mots, 